\title{
Adherent Diamond-Like Carbon Coatings on Metals Via PECVD and IBAD
}

\author{
Gil Capote*, Luís F. Bonetti ${ }^{* \dagger}$, Lúcia V. Santos*, Vladimir J. Trava-Airoldi*, and Evaldo J. Corat* \\ * Laboratório Associado de Sensores e Materiais, Instituto Nacional de Pesquisas Espaciais, \\ Caixa Postal 515, 12245-970, São José dos Campos - SP, Brazil \\ ${ }^{\dagger}$ Instituto Tecnológico de Aeronáutica, Centro Técnico Aeroespacial, São José dos Campos - SP, Brazil
}

\section{Received on 8 December, 2005}

\begin{abstract}
Adherent and low-stress a-C:H films were deposited on Ti6Al4V and stainless steel substrates using PECVD and IBAD techniques. An amorphous silicon interlayer was applied to improve the adhesion of the a-C:H films on the metal substrates. The XPS technique was employed to analyze the chemical bondings within the interfaces. The elemental composition and atomic density of the films were determined by ion beam analysis. The film microstructure was studied by means of Raman scattering spectroscopy. The mechanical properties were determined by means of stress and hardness measurements. The adherence was evaluated by means of scratch tests. The tests showed that the composition, the microstructure, and the mechanical properties of the films depend on the intensity of the ion bombardment and on the ion current.
\end{abstract}

Keywords: Diamond-like carbon; Plasma enhanced chemical vapor deposition; Ion beam-assisted deposition

\section{INTRODUCTION}

Research and development of nanostructured carbon-based coatings with tailor-designed properties is a fundamental need for the automotive, aerospace, biomedical and electronic industries. The amorphous hydrogenated carbon $(\mathrm{a}-\mathrm{C}: \mathrm{H})$ family of films stands out due to their properties of high hardness, chemical inertness, low friction and high-wear resistance. The major disadvantage of super hard diamond-like carbon (DLC) film deposition and, therefore, their technical applications is that there is often a relatively low adhesion of these films on metallic substrates caused by very high total compressive stress on these coatings. To overcome the low adhesion problems of these films on metallic substrates, different coating concepts have been proposed by many research groups, such as: deposition of a thin metal interlayer [1-2]; surface implantation [3]; chemical interlayer gradients or a multilayer coating [4]; variation of the self-bias voltage in the beginning of the deposition [5]; and use of surface thermal treatments [6]. The interlayers, especially the multilayers, cause a continual change in the thermal expansion coefficient and help to reduce stress in the a-C:H films.

DLC film deposition can be accomplished economically at low temperature, utilizing plasma enhanced chemical vapor deposition (PECVD) techniques, sputtering methods, arcdischarge, pulsed laser deposition (PLD), and ion beamassisted deposition (IBAD) techniques [7]. The adherent and low-stress a-C:H films are mostly produced by plasma decomposition of a hydrocarbon-containing precursor atmosphere using PECVD methods. Different silicon precursors have been applied to deposit a thin silicon interlayer between metallic substrates and a-C:H films in order to increase adherence of the coatings $[1,2,8]$. The IBAD, a combination of ion implantation and physical vapor deposition, can utilize energetic ion bombardment to enhance the films' adhesion on metals and to deposit the films at low substrate temperature. The IBAD process probably offers the only means to deposit DLC films onto steels and others metal substrates with particularly good interface adhesion [9-11].
In this study, both PECVD and IBAD systems were used to deposit adherent and low-stress a-C:H films on Ti6Al4V and stainless steel substrates. A thin amorphous silicon interlayer was used to improve the a-C:H films' adhesion. The films deposited via PECVD and IBAD were analyzed according to their microstructure, mechanical and tribological properties as a function of self-bias voltage and ion current.

\section{EXPERIMENTAL PROCEDURES}

DLC films were deposited by the PECVD and the IBAD methods, employing an asymmetrical capacitively-coupled deposition system [7] and end-Hall ion source [11], respectively. The thin amorphous silicon interlayers (10-100 $\mathrm{nm}$ ) were deposited only by the PECVD technique, which uses silane as the precursor to improve the film's adhesion. Ti6Al4V alloy and stainless steel 304 substrates were polished by using up to 0.25 microns of diamond powder and were cleaned ultrasonically in an acetone bath before putting them into a vacuum chamber. The substrates were additionally cleaned in argon discharge prior to deposition. In addition, $\mathrm{Si}$ (100) substrates were used in order to measure the total stress of the a-C:H films. The films obtained by r.f. PECVD were deposited with a total gas pressure of $10.5 \mathrm{~Pa}$ at 5 and $10 \mathrm{sccm}$ gas flows for silane and methane, respectively. The self-bias voltage was varied from 0 up to $-500 \mathrm{~V}$ according to growth parameters by adjusting the r.f. power input for both silicon and DLC films.

In the IBAD method, argon was used as the bombardment gas, while methane was used as a precursor. The argon/methane rate was kept constant at $1 / 4$, while the ion energy was maintained constant at $150 \mathrm{eV}$. The operating pressure in the chamber was approximately $3 \times 10^{-4} \mathrm{~Pa}$. A series of DLC films were deposited by changing the ion current from 2 up to $4.5 \mathrm{~A}$. Film thickness in the range of approximately 2 $\mu \mathrm{m}$ was determined by stylus profilometry.

X-ray photoelectron spectroscopy (XPS) was employed to investigate the chemical bondings within the interfaces, us- 
ing a surface Kratos XSAM HS instrument with monochromatic Al K $\alpha$ line of an X-ray source with energy of 1486.6 $\mathrm{eV}$ and $168 \mathrm{~W}$ of power. The measurements were taken in an ultra-high vacuum environment. Binding energy positions were verified using the $\mathrm{C} 1 \mathrm{~s}$ peak at $284.8 \mathrm{eV}$, which was associated to $\mathrm{C}-\mathrm{C}$ and/or $\mathrm{C}-\mathrm{H}$ bindings. The peaks were adjusted using Gaussian curves and the background was approximated by the Shirley method.

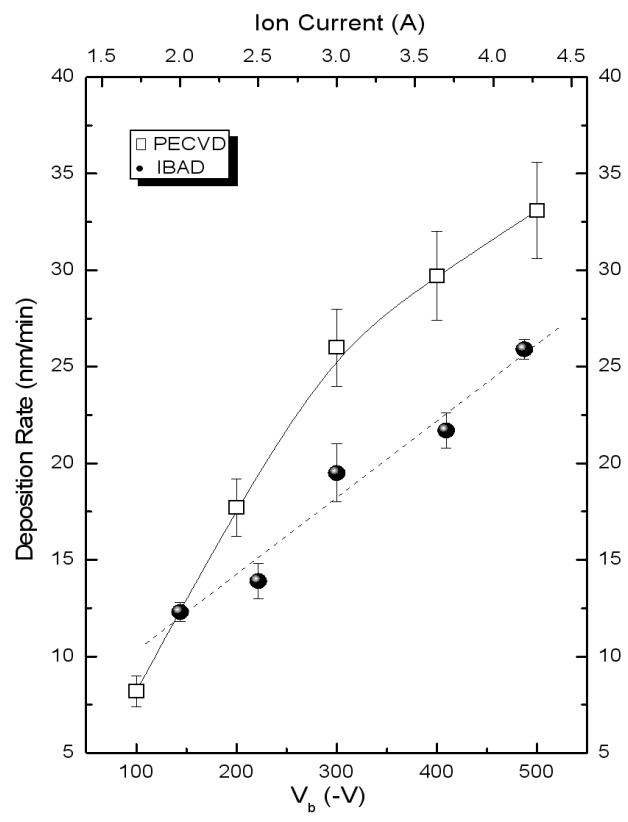

FIG. 1: Deposition rate as a function of the $\mathrm{V}_{b}$ and of the $\mathrm{I}_{c}$ for films deposited by PECVD and IBAD, respectively.

The elemental composition was determined by ion beam analysis (IBA), using Rutherford backscattering spectrometry (RBS) and elastic recoil detection analysis (ERDA) to determine the relative concentration of hydrogen, employed a 1.6-MV Pelletron electrostatic accelerator 5SDH from the National Electrostatic Corporation. For the measurements, a 2.2$\mathrm{MeV} \mathrm{He}^{+}$beam was used. The atomic density was inferred by combining the atomic density of the area provided by IBA and the film thickness.

The film's atomic arrangements were analyzed by Raman scattering spectroscopy. The spectroscopy was performed with a Renishaw 2000 system using an $\mathrm{Ar}^{+}$-ion laser $(\lambda=$ $514 \mathrm{~nm}$ ) in backscattering geometry. The laser power on the sample was $\sim 0.6 \mathrm{~mW}$ and the laser spot had a $2.5 \mu \mathrm{m}$ diameter. The Raman shift was calibrated in relation to the diamond pick at $1332 \mathrm{~cm}^{-1}$. All measurements were carried out in air at room temperature.

Total stress was determined by measuring the film curvature by means of stylus profilometry and by applying Stoney's
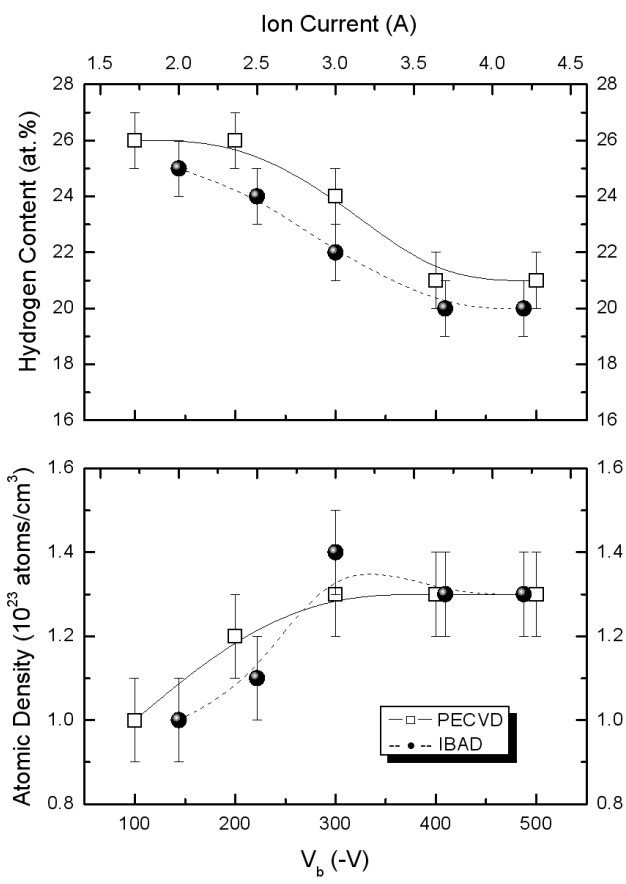

FIG. 2: The hydrogen content and atomic density of the a-C:H films as functions of the $V_{b}$ and of the $I_{c}$.

equation [12]. The hardness of the films was measured by employing a Fisherscope micro indenter, applying a load of $20 \mathrm{mN}$. The values presented in this study correspond to the average of 13 indentations.

Micro-scratch tests were used to evaluate the film adherence, using a conical tip, $200 \mu \mathrm{m}$ radius, diamond stylus (Rockwell C $120^{\circ}$ ). The normal force was kept constant for each test at 5,10 , and $20 \mathrm{~N}$.

\section{RESULTS AND DISCUSSIONS}

From XPS spectra, the presence of $\mathrm{C} 1 \mathrm{~s}, \mathrm{O} 1 \mathrm{~s}, \mathrm{Si} 2 \mathrm{~s}$ and Si $2 p$ peaks were observed. Oxygen may have been incorporated into the films from residual water pressure inside the chamber and from the film's exposure to the laboratory atmosphere. The results suggest that $\mathrm{SiC}(\sim 25 \%$ of the silicon peak) formed in the interface between the amorphous silicon interlayer and DLC films. This chemical bonding is strong and it is probably responsible for the good DLC film adherence on the silicon layer. Most of the silicon appeared in metallic form $\left(\mathrm{Si}^{0}\right)(\sim 55 \%)$, which is probably responsible for the silicon binding with the metallic substrate. In the DLC films, C-C and C-H bindings predominated $(\sim 70 \%)$. This result was expected since these chemical bondings are the majority in a-C:H films.

Figure 1 shows the deposition rate as a function of the selfbias voltage $\left(\mathrm{V}_{b}\right)$ and as a function of the ion current $\left(\mathrm{I}_{c}\right)$ for 

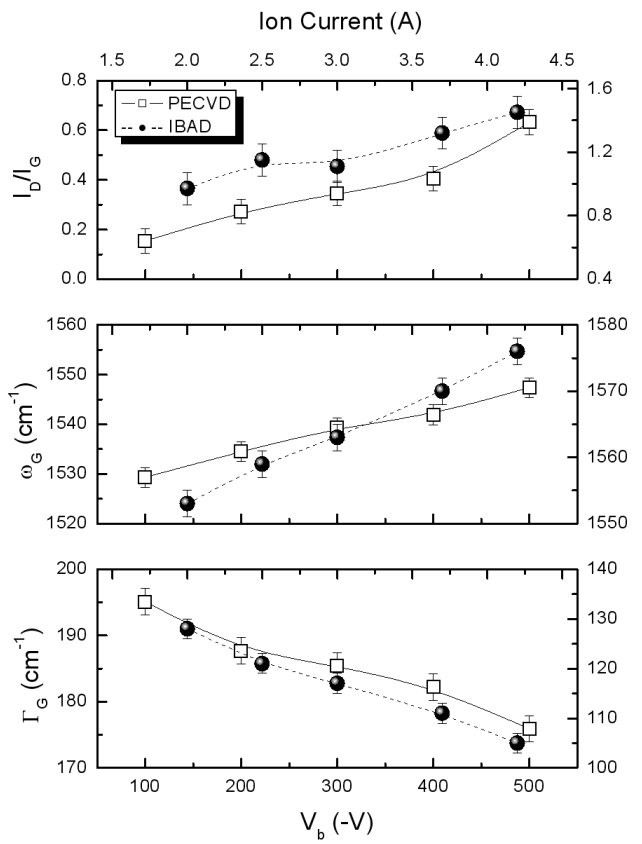

FIG. 3: $I_{D} / I_{G}$ intensity ratio, $\mathrm{G}$ band peak position $\left(\omega_{G}\right)$ and $\mathrm{G}$ bandwidth $\left(\Gamma_{G}\right)$ as a function of the $\mathrm{V}_{b}$ and $\mathrm{I}_{c}$.

films deposited by PECVD and IBAD, respectively. In both cases, increases in the deposition rates were observed. This behavior can be explained by the higher power input needed to achieve the desired self-bias voltage and consequently, the more efficient plasma dissociation. Films deposited by IBAD method needed, on the other hand, a higher methane flow to obtain the higher values of the ion current. Higher intensity ion bombardment may have contributed to the increased number of absorption sites.

The hydrogen content and atomic density of the a-C:H films as a function of the $\mathrm{V}_{b}$ and of the $\mathrm{I}_{c}$ are presented in Fig. 2. In both cases, the films deposited at low $\mathrm{V}_{b}$ and $\mathrm{I}_{c}$ presented higher hydrogen contents and lower atomic densities, while for higher $\mathrm{V}_{b}$ and $\mathrm{I}_{c}$ values, the density increased and hydrogen content decreased. The hydrogen content and the atomic density kept approximately constant values (21 at.\% and $1.3 \times 10^{23}$ atoms $/ \mathrm{cm}^{3}$, respectively). These values correspond to what are typically reported in the literature for a$\mathrm{C}: \mathrm{H}$ films [7]. This behavior is due to a more energetic ion bombardment, leading to preferential hydrogen sputtering that occurs because of the weaker strength of $\mathrm{C}-\mathrm{H}$ bonds when compared to the $\mathrm{C}-\mathrm{C}$ bonds. Moreover, greater $\mathrm{CH}_{4}$ molecule fragmentation in the plasma as the power was increased may have contributed to the decrease of the incorporation of hydrogen.

The Raman spectra for a-C:H films presented two overlapping bands known as D and $\mathrm{G}$ bands, which appear approximately at $1390 \mathrm{~cm}^{-1}$ and at $1545 \mathrm{~cm}^{-1}$, respectively. The spectra can be fitted using two Gaussian lines. The $I_{D} / I_{G}$ intensity ratio, $\mathrm{G}$ band peak position $\left(\omega_{G}\right)$ and $\mathrm{G}$ bandwidth $\left(\Gamma_{G}\right)$ as a function of the $\mathrm{V}_{b}$ and $\mathrm{I}_{c}$, obtained from the fitted parameters, for films deposited by both techniques are plotted in Fig. 3. The increase of the $I_{D} / I_{G}$ ratio, together with the shift of the $G$ band's peak position towards higher frequencies, accompanied by a reduction of the bandwidth, is usually interpreted in terms of an increase of graphitic domains, either in number or in size [13]. The results suggest a progressive graphitization of the a-C:H films upon self-bias and ion current increases.

Figures 4 and 5 present the total compressive stress and the hardness values as functions of the self-bias voltage and of the ion current, respectively. In Fig. 4, the stress measurements for a-C:H films deposited by PECVD directly on $\mathrm{Si}(100)$ substrate, on metallic substrate with amorphous silicon interlayer, and for films deposited by IBAD are presented. A significant decrease of the stress values was observed when the a-C:H films were deposited using a silicon interlayer. This result suggests that internal stress reduction is responsible for the increase of a-C:H film adherence on metallic substrates. Also, a significant decrease of the stress values for the film deposited by IBAD was observed. Both the internal stress and hardness values reached a maximum. The existence of this maximum in a-C:H films has been explained by the subplantation model [14]. The stress and hardness reduction in the films deposited with higher $\mathrm{V}_{b}$ and $\mathrm{I}_{c}$ suggests a progressive graphitization, as had been observed in the Raman results.

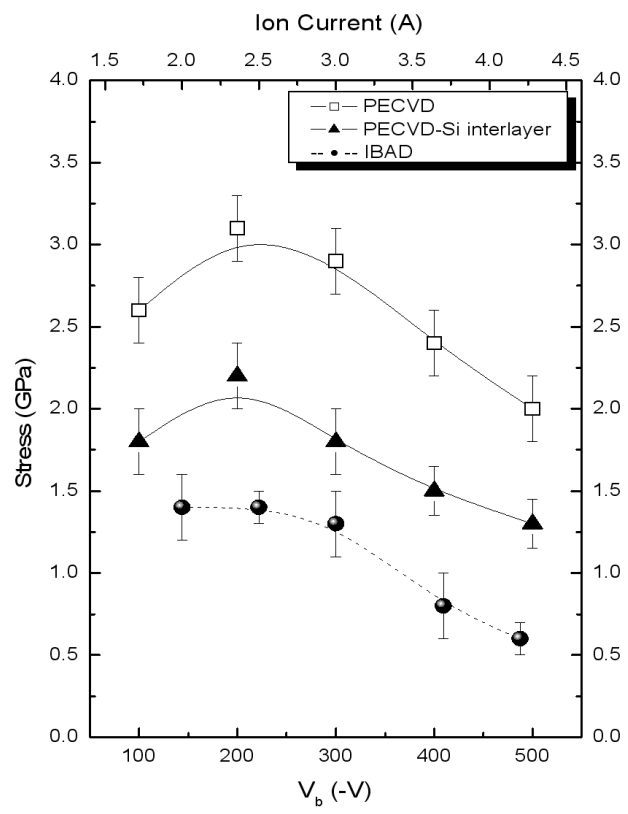

FIG. 4: Total compressive stress as a function of the $\mathrm{V}_{b}$ and $\mathrm{I}_{c}$ for films deposited by both techniques.

Micro-scratch tests showed that the coatings only cracked in a roughly semicircular arc along the scratch, without peeling off when the $20 \mathrm{~N}$ loads were applied. These results suggest strong DLC film adhesion on these substrates when the silicon interlayer was used. 


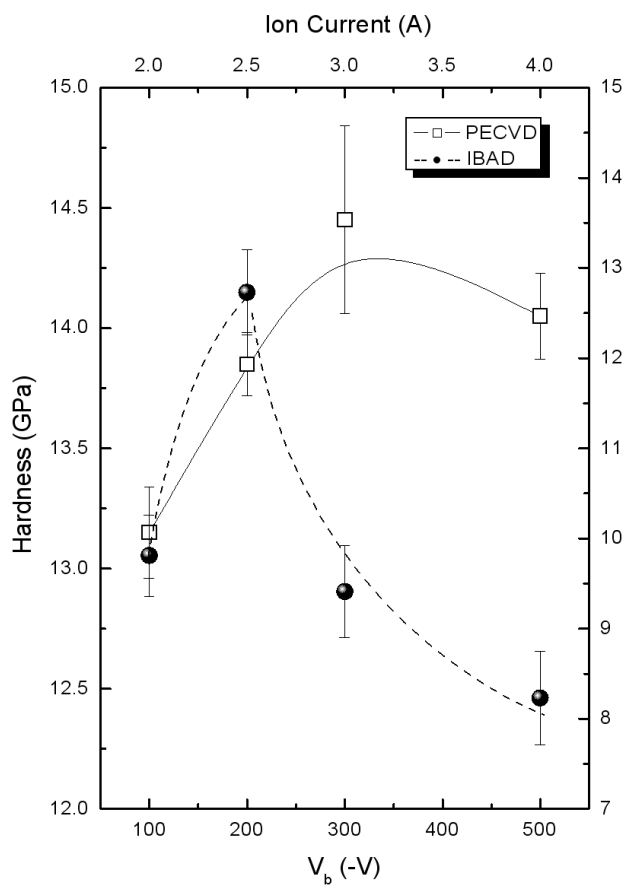

FIG. 5: Hardness as a function of the $\mathrm{V}_{b}$ and $\mathrm{I}_{c}$ for a-C:H films deposited by PECVD and IBAD techniques.

\section{CONCLUSION}

The use of the thin amorphous silicon interlayer enhanced the deposition of adherent, low-stress a-C:H films on Ti6Al4V and stainless steel substrates via PECVD. The $\mathrm{V}_{b}$ for PECVD and $\mathrm{I}_{c}$ for IBAD techniques play a similar role in term of total stress, hardness, and adherence studies. Analysis of DLC film showed that the composition, the microstructure, and the mechanical properties of these films depend on the intensity of ion bombardment and on the ion current.

A comparison of the results obtained with the PECVD and the IBAD techniques shown the similar results for the deposition rate, hydrogen content, atomic density, and Raman parameters. A decrease of the total compressive stress and of the hardness values were observed in the films deposited by IBAD method.

\section{Acknowledgement}

This study was supported by FAPESP and CNPq.
[1] V. Bursikova, V. Navratil, L. Zajickova, and J. Janca, Mater. Sci. Eng. A324, 251 (2002).

[2] S. Takeuchi, A. Tanji, H. Miyazawa, and M. Murakawa, Thin Solid Films 447-448, 208 (2004).

[3] C.S. Lee, T.Y. Kim, K.R. Lee, and K.H. Yoon, Thin Solid Films 447-448, 169 (2004)

[4] Q.R. Hou, J. Gao, Appl. Phys. A 68(3), 343 (1999).

[5] S. Zhang, X.L. Bui, Y. Fu, D.L. Butler, and H. Du, Diamond Relat. Mater. 13, 867 (2004).

[6] P. Mosaner, M. Bonelli, and A. Miotello, Appl. Surf. Sci. 208209, 561 (2003).

[7] J. Robertson, Mater. Sci. Eng. R37, 129 (2002).

[8] Y. Jun, J.Y. Choi, K.R. Lee, B.K. Jeong, S.K. Kwon, and Ch.H.
Hwang, Thin Solid Films 377-378, 233 (2000).

[9] J. Wang, W.Z. Li, and H.D. Li, Surf. Coat. Technol. 122, 273 (1999).

[10] Q. Jun, L. Jianbin, W. Shizhu, W. Jing, and L. Wenzhi, Surf. Coat. Technol. 128-129, 324 (2000).

[11] H.R. Kaufman, R.S. Robinson, and R.I. Seddon, J. Vac. Sci. Technol. A5 (4) 2081 (1987).

[12] G.G. Stoney, Proc. Royal Soc. London Series A 82, 172 (1909).

[13] O. Dillon, J. Woollam, and V. Katakana, Phys. Rev. B29, 3482 (1984).

[14] Y. Lifshitz, S.R. Kasi, J.W. Rabalais, and W. Eckstein, Phys. Rev. B 41, 10468 (1990). 